\title{
Childhood generalized specific phobia as an early marker of internalizing psychopathology across the lifespan: results from the World Mental Health Surveys
}

Ymkje Anna de Vries ${ }^{1,2^{*}}$, Ali Al-Hamzawi ${ }^{3}$, Jordi Alonso ${ }^{4,5,6}$, Guilherme Borges ${ }^{7}$, Ronny Bruffaerts ${ }^{8}$, Brendan Bunting ${ }^{9}$, José Miguel Caldas-de-Almeida ${ }^{10}$, Alfredo H. Cia ${ }^{11}$, Giovanni De Girolamo ${ }^{12}$, Rumyana V. Dinolova ${ }^{13}$, Oluyomi Esan ${ }^{14}$, Silvia Florescu ${ }^{15}$, Oye Gureje ${ }^{16}$, Josep Maria Haro ${ }^{17,18}$, Chiyi Hu ${ }^{19}$, Elie G. Karam 20,21,22, Aimee Karam²2, Norito Kawakamii ${ }^{23}$, Andrzej Kiejna ${ }^{24,25}$, Viviane Kovess-Masfety ${ }^{26}$, Sing Lee ${ }^{27}$, Zeina Mneimneh ${ }^{28}$, Fernando Navarro-Mateu ${ }^{29,30,31}$, Marina Piazza ${ }^{32,33}$, Kate Scott ${ }^{34}$, Margreet ten Have ${ }^{35}$, Yolanda Torres ${ }^{36}$, Maria Carmen Viana ${ }^{37}$, Ronald C. Kessler ${ }^{38}$, Peter de Jonge ${ }^{1,2}$ and on behalf of the WHO World Mental Health Survey Collaborators

\footnotetext{
Abstract

Background: Specific phobia (SP) is a relatively common disorder associated with high levels of psychiatric comorbidity. Because of its early onset, SP may be a useful early marker of internalizing psychopathology, especially if generalized to multiple situations. This study aimed to evaluate the association of childhood generalized SP with comorbid internalizing disorders.

Methods: We conducted retrospective analyses of the cross-sectional population-based World Mental Health Surveys using the Composite International Diagnostic Interview. Outcomes were lifetime prevalence, age of onset, and persistence of internalizing disorders; past-month disability; lifetime suicidality; and 12-month serious mental illness. Logistic and linear regressions were used to assess the association of these outcomes with the number of subtypes of childhood-onset ( $<13$ years) SP.

(Continued on next page)
}

\footnotetext{
* Correspondence: y.a.de.vries@rug.nl

${ }^{1}$ Faculty of Behavioural and Social Sciences, Department of Developmental

Psychology, University of Groningen, Groningen, the Netherlands

${ }^{2}$ Interdisciplinary Center Psychopathology and Emotion regulation, University

Medical Center Groningen, University of Groningen, Groningen, the

Netherlands

Full list of author information is available at the end of the article
}

(c) The Author(s). 2019 Open Access This article is distributed under the terms of the Creative Commons Attribution 4.0 International License (http://creativecommons.org/licenses/by/4.0/), which permits unrestricted use, distribution, and reproduction in any medium, provided you give appropriate credit to the original author(s) and the source, provide a link to the Creative Commons license, and indicate if changes were made. The Creative Commons Public Domain Dedication waiver (http://creativecommons.org/publicdomain/zero/1.0/) applies to the data made available in this article, unless otherwise stated. 


\begin{abstract}
(Continued from previous page)
Results: Among 123,628 respondents from 25 countries, retrospectively reported prevalence of childhood SP was $5.9 \%, 56 \%$ of whom reported one, $25 \%$ two, $10 \%$ three, and $8 \%$ four or more subtypes. Lifetime prevalence of internalizing disorders increased from $18.2 \%$ among those without childhood SP to $46.3 \%$ among those with one and $75.6 \%$ those with $4+$ subtypes $(\mathrm{OR}=2.4,95 \% \mathrm{Cl} 2.3-2.5, p<0.001)$. Twelve-month persistence of lifetime internalizing comorbidity at interview increased from $47.9 \%$ among those without childhood SP to $59.0 \%$ and $79.1 \%$ among those with 1 and $4+$ subtypes (OR $=1.4,95 \% \mathrm{Cl} 1.4-1.5, p<0.001)$. Respondents with $4+$ subtypes also reported significantly more disability (3.5 days out of role in the past month) than those without childhood SP (1.1 days) or with only 1 subtype (1.8 days) $(B=0.56, \mathrm{SE} 0.06, p<0.001)$ and a much higher rate of lifetime suicide attempts (16.8\%) than those without childhood SP (2.0\%) or with only 1 subtype (6.5\%) $(\mathrm{OR}=1.7,95 \% \mathrm{Cl}$ $1.7-1.8, p<0.001)$.

Conclusions: This large international study shows that childhood-onset generalized SP is related to adverse outcomes in the internalizing domain throughout the life course. Comorbidity, persistence, and severity of internalizing disorders all increased with the number of childhood SP subtypes. Although our study cannot establish whether SP is causally associated with these poor outcomes or whether other factors, such as a shared underlying vulnerability, explain the association, our findings clearly show that childhood generalized SP identifies an important target group for early intervention.
\end{abstract}

Keywords: Specific phobia, Internalizing disorders, Early markers, Comorbidity, Suicidality,

\section{Introduction}

Anxiety and mood disorders are major contributors to the global disease burden [1] due partly to their high prevalence [2], early onset [3], and chronic or recurrent course [4-6]. Although much effort has been devoted to improving the course of these disorders, treatment is still insufficient to avert most of the burden [7-11]. Consequently, prevention may be a necessary alternative strategy.

Comorbidity between mental disorders is common $[12,13]$, which has led theorists to posit a latent structure of psychopathology, reducing a variety of disorders to a limited set of domains [14]. These domains, of which the internalizing and externalizing have been confirmed most frequently [15-20], are thought to represent core psychopathological processes underlying the varied clinical manifestations of disorders. If so, targeting these underlying processes might offer new opportunities for prevention. Internalizing disorders, for example, develop at very different ages: specific phobia (SP) has a median age of onset at 8 years old [21], whereas major depressive disorder (MDD) and generalized anxiety disorder (GAD) have median onset at 30-40 years old [3, 22]. If interventions could successfully treat the earlier disorders in this domain, this might lead to reductions in the subsequent onset, persistence, or severity of other disorders in the same domain.

Although SP is often considered a relatively mild disorder [23, 24], the structure of psychopathology model suggests that it might identify persons with a vulnerability for more serious later disorders in the same domain. In support of this possibility, SP has been associated with increased risk of later-onset disorders in the internalizing domain [21, 25-34]. Importantly, persons with SP may have only a single fear, or they may have many (e.g., of spiders, storms, blood, and heights). Some evidence suggests that persons with multiple fears have a greater risk of comorbidity and impairment $[21,29,33$, 35 , 36], suggesting that generalized SP may be a marker of particularly high internalizing vulnerability.

With an eye toward early intervention, SP with an onset in childhood is of greatest interest, but to date, no study has examined internalizing comorbidity in this group of people. Furthermore, information about other important aspects of comorbidity, such as age of onset, persistence, and severity, and other outcomes, such as suicidality, is largely lacking. In this paper, we therefore examine these outcomes. We focus on the number of phobia subtypes as a marker of generalization of the underlying psychopathological process, expecting worse outcomes among persons reporting more fears.

\section{Methods}

\section{Survey samples}

Data came from the World Mental Health Surveys (WMHS), which include cross-sectional surveys administered in low/lower-middle-income countries, uppermiddle-income countries, and high-income countries (Additional file 1: Table S1). Adults were selected using probability sampling methods designed to generate population representative samples, and interviews were conducted face to face in respondent homes. A total of 123,628 respondents from 25 countries participated in the present study. Informed consent was obtained according to protocols endorsed by local Institutional Review Boards. 
Within-country sampling methods are described in detail elsewhere [37, 38].

\section{Measures \\ Mental disorders}

Lifetime and 12-month mental disorders were assessed with the WHO Composite International Diagnostic Interview (CIDI) [39]. To reduce respondent burden, interviews were administered in two parts. All respondents completed part I, assessing core mental disorders. Part II, assessing other disorders and correlates, was administered to all respondents with any lifetime part I diagnosis and a probability subsample of other part I respondents. Part II data were weighted so that weighted prevalence estimates are identical to those in the part I sample.

We included the following internalizing disorders: anxiety disorders (panic disorder, agoraphobia, GAD, social anxiety disorder, SP, post-traumatic stress disorder [PTSD], separation anxiety disorder), mood disorders (MDD and/or dysthymia, bipolar disorder [I, II, or subthreshold]), and eating disorders (bulimia nervosa, binge eating disorder). CIDI diagnoses have shown generally good concordance with diagnoses based on blinded Structured Clinical Interview for DSM-IV (SCID) reappraisal [40]. Age of onset was assessed with retrospective reports. Persistence was defined as the presence of the disorder in the 12 months before interview among lifetime cases.

\section{Specific phobia}

The CIDI distinguishes between six SP subtypes: animals, still water or weather, high places, blood-injection-injury, closed spaces, and flying. For each participant, we determined how many subtypes with an age of onset prior to age 13 were reported. Because few participants reported more than four subtypes, we collapsed participants reporting four or more subtypes into a single group. Participants who developed SP later in life were included in the group of participants without childhood SP.

\section{Impairment and suicidality}

Severe role impairment due to SP was assessed with a modified Sheehan Disability Scale (SDS) [41]. Respondents with 12-month SP rated its interference with functioning in four domains (home management, work, close relationships, and social life) on a scale of 0-10 during the worst month in the past year. Severe impairment was defined as a score $\geq 7$ in at least one domain. Respondents with lifetime SP were asked whether they had ever sought treatment specifically for SP.

All respondents were asked how many days in the past 30 days they were totally unable to work or carry out normal activities because of any physical or mental health problems, whether they had ever seriously thought about committing suicide, and, if so, whether they had ever made a plan or attempted suicide. We also examined serious mental illness (SMI), which was defined as meeting criteria for 12-month bipolar I disorder or having another 12-month mood or anxiety disorder (other than SP) with either severe role impairment or a past-year suicide attempt [42].

\section{Statistical analysis}

We tested the linear association of the number of early-onset SP subtypes, both within the whole sample and within the subsample with childhood SP, using logistic regression for dichotomous variables and linear regression for continuous variables (SAS 9.4). We used the actuarial method to determine the age of onset of comorbid disorders and tested for differences in age of onset depending on the number of SP subtypes using discrete-time logistic regression in the subsample with the comorbid disorder. We also used the actuarial method to calculate the projected lifetime risk of any internalizing disorder, which takes into account that respondents who have not had a disorder yet may still develop the disorder later in life. We also estimated population attributable fractions (PAFs) [43], which indicate the fraction of an outcome in the population that is attributable to childhood SP (assuming a causal relationship, noting that the latter can only be confirmed experimentally). All analyses controlled for country of origin of the participant. Because the data were clustered and weighted, standard errors were estimated using the Taylor series linearization method (SUDAAN 11.0.1). Significance tests were evaluated using $\alpha=0.005$ (twosided) to reduce the chance of false-positive findings given the many tests performed.

As sensitivity analyses, we tested interactions between the number of SP subtypes and age group (18-34, 3549 , and $50+$ years old), to examine whether associations between childhood phobia and mental health outcomes persisted into older adulthood. We also tested whether associations were found for participants with and without current internalizing psychopathology and for participants from high-income countries as well as those from low- or middle-income countries.

\section{Results}

\section{Specific phobia}

Out of 123,628 participants, $51.8 \%$ were female and the mean age was $42.0(\mathrm{SD}=16.9)$. Lifetime and 12-month SP prevalence was $7.6 \%$ and $5.7 \%$, respectively. Most respondents reported onset before the age of 13, resulting in a $5.9 \%$ lifetime prevalence of childhood-onset SP. Prevalence was $3.3 \%$ for one early-onset subtype, $1.5 \%$ for 
two, $0.6 \%$ for three, and $0.5 \%$ for $4+$ (Table 1 ). Persistence was high and increased with number of subtypes (from $73.4 \%$ for participants with one subtype to $85.2 \%$ for those with $4+$ subtypes, $\mathrm{OR}=1.2, p<0.001)$. Severe impairment due to SP and treatment for SP were uncommon (17.5$26.5 \%)$, but increased with an increasing number of subtypes $(\mathrm{OR}=1.1-1.3, p=0.004$ and $<0.001)$.

\section{Internalizing disorder comorbidity}

Lifetime prevalence of any internalizing disorder increased from $18.2 \%$ among those without childhood SP to $46.3 \%$ among those with one SP subtype and $75.6 \%$ among those with $4+$ subtypes $(\mathrm{OR}=2.4$ in the total sample and 1.6 in the SP subsample, $p<0.001$ ) (Table 2). Figure 1 shows the projected risk of any internalizing disorder by age and number of SP subtypes. Examining separate disorder groupings, similar patterns were apparent for anxiety disorders $(\mathrm{OR}=2.4$ (total sample) and 1.6 (SP subsample), $p<0.001)$, mood disorders $(\mathrm{OR}=1.9$ (total sample) and 1.4 (SP subsample), $p<0.001$ ), and eating disorders $(\mathrm{OR}=1.8$ (total sample) and 1.3 (SP subsample), $p<0.001$ and $p=0.003$ ). Only $0.6 \%$ of respondents without SP and $4.2 \%$ of those with one SP subtype met criteria for $4+$ internalizing disorders (other than SP), compared to $19.4 \%$ of respondents with $4+$ SP subtypes $(\mathrm{OR}=2.6$ (total sample) and 1.7 (SP subsample), $p<0.001)$. SP preceded other internalizing disorders in $79.4 \%$ of comorbid cases; in an additional $10.8 \%$ of cases, the onset of SP and another internalizing disorder coincided.

Estimated PAFs for any childhood SP ranged from 8.7\% for MDD to $38.8 \%$ for agoraphobia (Additional file 1: Table S2). This means that if the associations found here are accurate reflections of causal effects either of childhood SP or of a latent liability that could be successfully treated by early intervention with childhood SP, the population-level lifetime prevalence of these disorders would be expected to decrease proportionally by $8.7-$ $38.8 \%$. The PAFs for any internalizing disorder and for $4+$ internalizing disorders were $10.2 \%$ and $39.4 \%$, respectively.

Persistence of any lifetime internalizing disorder increased from $47.9 \%$ for respondents without childhood SP to $59.0 \%$ for those with one SP subtype and $79.1 \%$ for those with $4+$ subtypes (OR $=1.4$ (total sample) and 1.3 (SP subsample), $p<0.001$ ) (Table 3 ). Examining separate disorder groupings, similar patterns were found for anxiety disorders $(\mathrm{OR}=1.4$ (total sample) and 1.2 (SP subsample), $p<0.001$ ) and mood disorders $(\mathrm{OR}=1.3$ (total sample) and 1.2 (SP subsample), $p<0.001$ ), but not for eating disorders $(\mathrm{OR}=1.1$ (total sample) and 0.8 (SP subsample), $p=0.164-0.295)$.

Median age of onset of comorbid internalizing disorders generally decreased with increasing number of early-onset SP subtypes (Table 4), especially for agoraphobia, GAD, panic disorder, and MDD/dysthymia. For GAD, median age of onset was 39 for those without childhood SP, 37 for those with one subtype, and 28 for those with $4+$ subtypes $(\mathrm{OR}=1.2$ (total sample and SP subsample), $p<0.001)$. Similarly, median age of onset decreased from 21 to 12 for agoraphobia, from 33 to 18 for panic disorder, and from 38 to 29 for MDD/dysthymia $(\mathrm{OR}=1.2$ (total sample) and 1.1-1.3 (SP subsample), $p<0.001)$. For social anxiety disorder, there was also a consistent, though slight, decrease in age of onset with an increasing number of SP subtypes (from 14 to 12 , $\mathrm{OR}=1.2$ (total sample) and 1.3 (SP subsample), $p<$ 0.001). For PTSD, separation anxiety disorder, and bipolar disorder, patterns were less consistent $(\mathrm{OR}=1.0-1.2$, $p<0.001-0.235)$.

\section{Impairment, suicidality, and serious mental illness}

Respondents without childhood SP reported a mean (SE) of 1.05 (0.03) days out of role in the past 30 days, compared to 1.80 (0.19) days among respondents with one SP subtype and $3.53(0.53)$ days among those with 4 + subtypes $(B=0.56$ (total sample) and 0.49 (SP subsample), $p<0.001$ ) (Table 5). Suicidality was also relatively common among those with childhood SP, with

Table 1 Prevalence and characteristics of specific phobia

\begin{tabular}{|c|c|c|c|c|c|c|c|c|}
\hline & \multirow{2}{*}{$\begin{array}{l}\text { Any } \\
\text { specific } \\
\text { phobia }\end{array}$} & \multirow{2}{*}{$\begin{array}{l}\text { Any } \\
\text { early- } \\
\text { onset } \\
\text { specific } \\
\text { phobia }\end{array}$} & \multicolumn{4}{|c|}{ Number of subtypes } & \multicolumn{2}{|c|}{ Test of linear effect } \\
\hline & & & 1 & 2 & 3 & $4+$ & & \\
\hline & $\%(\mathrm{SE})$ & $\%(\mathrm{SE})$ & $\%(\mathrm{SE})$ & $\%(\mathrm{SE})$ & $\%(\mathrm{SE})$ & $\%(\mathrm{SE})$ & OR (95\% Cl) & $p$ value \\
\hline Lifetime prevalence & $7.6(0.1)$ & $5.9(0.1)$ & $3.3(0.1)$ & $1.5(0.0)$ & $0.6(0.0)$ & $0.5(0.0)$ & - & - \\
\hline 12-month prevalence & $5.7(0.1)$ & $4.4(0.1)$ & $2.4(0.1)$ & $1.1(0.0)$ & $0.5(0.0)$ & $0.4(0.0)$ & - & - \\
\hline Persistence & $74.3(0.6)$ & $75.2(0.7)$ & $73.4(0.9)$ & $75.2(1.3)$ & $77.6(1.8)$ & $85.2(1.8)$ & $1.2^{*}(1.1-1.3)$ & $<0.001$ \\
\hline Severe disability & $19.1(0.6)$ & $19.7(0.6)$ & $17.5(0.8)$ & $20.2(1.4)$ & $24.0(2.0)$ & $26.5(2.4)$ & $1.3^{*}(1.1-1.4)$ & $<0.001$ \\
\hline Treatment for specific phobia & $22.4(0.5)$ & $20.8(0.6)$ & $19.1(0.8)$ & $22.9(1.2)$ & $22.4(1.7)$ & $22.8(2.0)$ & $1.1^{*}(1.0-1.2)$ & 0.004 \\
\hline
\end{tabular}


Table 2 Prevalence of comorbid internalizing disorders, as a function of the number of specific phobia subtypes

\begin{tabular}{|c|c|c|c|c|c|c|c|c|c|}
\hline \multirow[t]{3}{*}{ Comorbid disorder } & \multicolumn{5}{|c|}{ Number of subtypes } & \multirow{2}{*}{\multicolumn{2}{|c|}{$\begin{array}{l}\text { Test of linear effect (total } \\
\text { sample) }\end{array}$}} & \multirow{2}{*}{\multicolumn{2}{|c|}{$\begin{array}{l}\text { Test of linear effect (SP } \\
\text { cases only) }\end{array}$}} \\
\hline & \multirow{2}{*}{$\begin{array}{l}0 \\
\%(S E)\end{array}$} & \multirow{2}{*}{$\begin{array}{l}1 \\
\%(\mathrm{SE}) \\
\end{array}$} & \multirow{2}{*}{$\begin{array}{l}2 \\
\%(\mathrm{SE}) \\
\end{array}$} & \multirow{2}{*}{$\begin{array}{l}3 \\
\%(\mathrm{SE})\end{array}$} & \multirow{2}{*}{$\begin{array}{l}4+ \\
\% \text { (SE) }\end{array}$} & & & & \\
\hline & & & & & & OR $(95 \% \mathrm{Cl})$ & $p$ value & OR $(95 \% \mathrm{Cl})$ & $p$ value \\
\hline Agoraphobia & $0.8(0.0)$ & $5.1(0.4)$ & $9.5(0.8)$ & $17.4(2.0)$ & $21.2(2.3)$ & $2.6^{*}(2.4-2.7)$ & $<0.001$ & $1.7^{*}(1.5-1.9)$ & $<0.001$ \\
\hline Generalized anxiety disorder & $3.5(0.1)$ & $9.7(0.6)$ & $14.2(1.1)$ & $21.0(1.8)$ & $21.3(2.6)$ & $1.8^{*}(1.7-1.9)$ & $<0.001$ & $1.4^{*}(1.3-1.5)$ & $<0.001$ \\
\hline Panic disorder & $1.4(0.0)$ & $5.5(0.5)$ & $8.8(0.7)$ & $12.6(1.5)$ & $15.8(1.8)$ & $2.0^{*}(1.9-2.1)$ & $<0.001$ & $1.5^{*}(1.3-1.6)$ & $<0.001$ \\
\hline Post-traumatic stress disorder & $2.9(0.1)$ & $9.0(0.6)$ & $15.3(1.2)$ & $18.5(1.8)$ & $22.2(2.3)$ & $1.9^{*}(1.8-2.0)$ & $<0.001$ & $1.4^{*}(1.3-1.6)$ & $<0.001$ \\
\hline Separation anxiety disorder & $3.9(0.1)$ & $12.7(0.9)$ & $19.7(1.7)$ & $27.2(2.5)$ & $27.4(2.7)$ & $1.9^{*}(1.8-2.0)$ & $<0.001$ & $1.4^{*}(1.2-1.5)$ & $<0.001$ \\
\hline Social anxiety disorder & $3.3(0.1)$ & $14.9(0.8)$ & $23.2(1.3)$ & $31.3(2.3)$ & $38.2(2.8)$ & $2.3^{*}(2.2-2.4)$ & $<0.001$ & $1.5^{*}(1.4-1.6)$ & $<0.001$ \\
\hline Any anxiety disorder & $10.5(0.2)$ & $33.6(1.1)$ & $47.0(1.7)$ & $62.4(2.3)$ & $66.1(2.8)$ & $2.4^{*}(2.3-2.5)$ & $<0.001$ & $1.6^{*}(1.5-1.7)$ & $<0.001$ \\
\hline Major depression/dysthymia & $10.5(0.2)$ & $24.1(0.9)$ & $30.1(1.4)$ & $36.2(2.5)$ & $34.1(2.7)$ & $1.6^{*}(1.6-1.7)$ & $<0.001$ & $1.2^{*}(1.2-1.3)$ & $<0.001$ \\
\hline Bipolar disorder & $1.8(0.1)$ & $5.7(0.5)$ & $10.0(0.9)$ & $12.7(1.5)$ & $17.3(1.9)$ & $1.9^{*}(1.8-2.0)$ & $<0.001$ & $1.5^{*}(1.3-1.6)$ & $<0.001$ \\
\hline Any mood disorder & $12.0(0.2)$ & $28.8(1.0)$ & $39.3(1.5)$ & $47.8(2.5)$ & $50.9(3.0)$ & $1.9^{*}(1.8-1.9)$ & $<0.001$ & $1.4^{*}(1.3-1.5)$ & $<0.001$ \\
\hline Bulimia nervosa & $0.6(0.0)$ & $2.0(0.3)$ & $3.6(0.7)$ & $6.8(1.6)$ & $5.9(1.6)$ & $2.0^{*}(1.8-2.2)$ & $<0.001$ & $1.7^{*}(1.4-2.0)$ & $<0.001$ \\
\hline Binge eating disorder & $1.4(0.1)$ & $5.7(0.8)$ & $5.3(0.8)$ & $6.6(1.7)$ & $6.3(1.7)$ & $1.7^{*}(1.5-1.8)$ & $<0.001$ & $1.0(0.8-1.3)$ & 0.746 \\
\hline Any eating disorder & $1.9(0.1)$ & $7.2(0.9)$ & $8.3(1.0)$ & $12.4(2.0)$ & $11.4(2.2)$ & $1.8^{*}(1.7-1.9)$ & $<0.001$ & $1.3^{*}(1.1-1.5)$ & 0.003 \\
\hline Any internalizing disorder & $18.2(0.2)$ & $46.3(1.2)$ & $61.1(1.7)$ & $75.6(2.1)$ & $75.6(2.7)$ & $2.4^{*}(2.3-2.5)$ & $<0.001$ & $1.6^{*}(1.5-1.8)$ & $<0.001$ \\
\hline Exactly 1 internalizing disorder & $12.3(0.2)$ & $24.0(1.0)$ & $27.6(1.5)$ & $26.8(2.2)$ & $21.6(2.4)$ & $1.4^{*}(1.3-1.4)$ & $<0.001$ & $1.0(0.9-1.1)$ & 0.677 \\
\hline Exactly 2 internalizing disorders & $4.0(0.1)$ & $12.2(0.7)$ & $15.2(1.2)$ & $21.6(2.0)$ & $19.7(2.2)$ & $1.7^{*}(1.6-1.8)$ & $<0.001$ & $1.2^{*}(1.1-1.3)$ & $<0.001$ \\
\hline Exactly 3 internalizing disorders & $1.4(0.0)$ & $5.9(0.5)$ & $9.2(0.8)$ & $12.8(1.5)$ & $14.9(1.8)$ & $2.0^{*}(1.9-2.1)$ & $<0.001$ & $1.4^{*}(1.2-1.5)$ & $<0.001$ \\
\hline $4+$ internalizing disorders & $0.6(0.0)$ & $4.2(0.4)$ & $9.0(0.8)$ & $14.4(1.7)$ & $19.4(2.6)$ & $2.6^{*}(2.4-2.8)$ & $<0.001$ & $1.7^{*}(1.6-2.0)$ & $<0.001$ \\
\hline
\end{tabular}

Italics indicate the main groups of disorders. ${ }^{*} p<0.005$

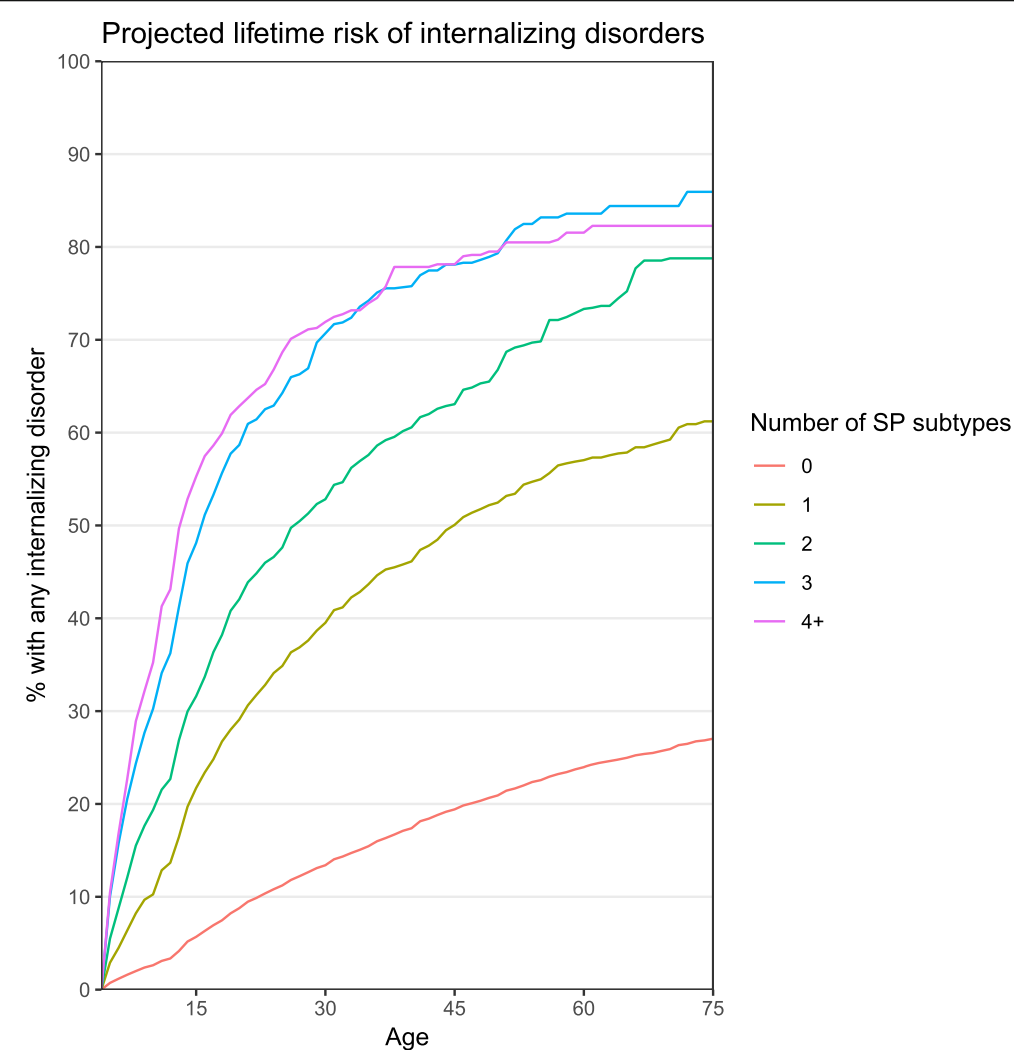

Fig. 1 Projected risk of any internalizing disorder by age 75, by number of childhood specific phobia subtypes. Projected risk was calculated using the actuarial method and accounts for censoring of participants who have not yet reached the age of 75 by the time of the interview 
Table 3 Persistence of comorbid internalizing disorders, as a function of the number of specific phobia subtypes

\begin{tabular}{|c|c|c|c|c|c|c|c|c|c|}
\hline \multirow[t]{3}{*}{ Comorbid disorder } & \multicolumn{5}{|c|}{ Number of subtypes } & \multirow{2}{*}{\multicolumn{2}{|c|}{$\begin{array}{l}\text { Test of linear effect (total } \\
\text { sample) }\end{array}$}} & \multirow{2}{*}{\multicolumn{2}{|c|}{$\begin{array}{l}\text { Test of linear effect (SP } \\
\text { cases only) }\end{array}$}} \\
\hline & \multirow{2}{*}{$\begin{array}{l}0 \\
\% \text { (SE) }\end{array}$} & \multirow{2}{*}{$\begin{array}{l}1 \\
\%(\mathrm{SE})\end{array}$} & \multirow{2}{*}{$\begin{array}{l}2 \\
\%(\mathrm{SE})\end{array}$} & \multirow{2}{*}{$\begin{array}{l}3 \\
\%(S E)\end{array}$} & \multirow{2}{*}{$\begin{array}{l}4+ \\
\%(S E)\end{array}$} & & & & \\
\hline & & & & & & OR $(95 \% \mathrm{Cl})$ & $p$ value & OR $(95 \% \mathrm{Cl})$ & $p$ value \\
\hline Agoraphobia & $57.8(2.6)$ & $67.6(3.9)$ & $64.6(5.1)$ & $63.6(6.6)$ & $86.4(3.2)$ & $1.3^{*}(1.1-1.4)$ & $<0.001$ & $1.3^{*}(1.1-1.6)$ & 0.002 \\
\hline Generalized anxiety disorder & $46.2(1.1)$ & $52.3(3.0)$ & $56.3(3.7)$ & $51.0(4.7)$ & $68.7(5.3)$ & $1.2^{*}(1.1-1.3)$ & $<0.001$ & $1.1(1.0-1.3)$ & 0.155 \\
\hline Panic disorder & $56.1(1.9)$ & $59.3(4.1)$ & $65.7(3.9)$ & $63.6(5.8)$ & $80.7(4.7)$ & $1.2^{*}(1.1-1.4)$ & $<0.001$ & $1.3^{*}(1.1-1.6)$ & 0.003 \\
\hline Post-traumatic stress disorder & $46.0(1.5)$ & $53.4(3.5)$ & $56.0(4.0)$ & $59.6(4.9)$ & $50.9(5.4)$ & $1.2^{*}(1.0-1.3)$ & 0.003 & $1.0(0.9-1.2)$ & 0.821 \\
\hline Separation anxiety disorder & $20.4(1.3)$ & $20.3(3.1)$ & $24.2(3.1)$ & $23.3(4.2)$ & $26.3(4.3)$ & $1.1(1.0-1.2)$ & 0.109 & $1.1(0.9-1.3)$ & 0.345 \\
\hline Social anxiety disorder & $56.2(1.3)$ & $65.3(2.7)$ & $73.9(2.8)$ & $73.4(3.6)$ & $80.2(3.5)$ & $1.3^{*}(1.2-1.5)$ & $<0.001$ & $1.2(1.0-1.3)$ & 0.027 \\
\hline Any anxiety disorder & $48.9(0.8)$ & $59.5(1.8)$ & $65.4(2.1)$ & $65.5(2.9)$ & $76.0(2.9)$ & $1.4^{*}(1.3-1.4)$ & $<0.001$ & $1.2^{*}(1.1-1.3)$ & $<0.001$ \\
\hline Major depression/dysthymia & $39.7(0.6)$ & $48.8(2.0)$ & $51.5(2.7)$ & $56.3(3.8)$ & $63.3(4.5)$ & $1.3^{*}(1.2-1.4)$ & $<0.001$ & $1.2(1.0-1.3)$ & 0.007 \\
\hline Bipolar disorder & $56.7(1.6)$ & $57.4(4.3)$ & $67.9(4.8)$ & $70.2(5.4)$ & $77.5(4.9)$ & $1.2^{*}(1.1-1.4)$ & $<0.001$ & $1.4^{*}(1.2-1.8)$ & 0.001 \\
\hline Any mood disorder & $41.9(0.5)$ & $50.2(1.8)$ & $55.3(2.4)$ & $59.7(3.1)$ & $68.0(3.3)$ & $1.3^{*}(1.2-1.4)$ & $<0.001$ & $1.2^{*}(1.1-1.4)$ & $<0.001$ \\
\hline Any eating disorder & $42.9(2.3)$ & $54.0(5.7)$ & $55.7(5.7)$ & $38.1(8.8)$ & $45.1(8.9)$ & $1.1(0.9-1.2)$ & 0.295 & $0.8(0.6-1.1)$ & 0.164 \\
\hline Any internalizing disorder & $47.9(0.5)$ & $59.0(1.5)$ & $66.1(2.0)$ & $70.5(2.5)$ & $79.1(2.5)$ & $1.4^{*}(1.4-1.5)$ & $<0.001$ & $1.3^{*}(1.2-1.4)$ & $<0.001$ \\
\hline
\end{tabular}

Persistence is defined as the presence of a 12-month disorder among lifetime cases with that disorder. Because of low prevalence, persistence of individual eating disorders was not assessed

Italics indicate the main groups of disorders. ${ }^{*} p<0.005$

$31.8 \%$ of those with $4+$ subtypes reporting lifetime suicidal ideation, compared to only $7.5 \%$ of those without childhood phobia and $18.8 \%$ of those with one SP subtype (OR $=1.6$ (total sample) and 1.3 (SP subsample), $p$ $<0.001$ ). Furthermore, $36.8 \%$ of respondents with $4+$ subtypes reported a 12-month SMI, compared to just $3.5 \%$ of those without childhood phobia and $12.7 \%$ of those with one subtype $(\mathrm{OR}=2.1$ (total sample) and 1.5 (SP subsample), $p<0.001$ ). PAFs for suicidality and SMI ranged from $13.9 \%$ for suicidal ideation to $20.4 \%$ for suicide attempts (Additional file 1: Table S3).

\section{Sensitivity analyses}

Associations of SP subtype number with prevalence, persistence, and severity of secondary comorbid disorders were generally consistent across age groups (Additional file 1: Table S4 and 5), suggesting that the associations described above are generally stable over the life course. Likewise, we found associations of SP subtype number with the lifetime prevalence of comorbid disorders and suicidality in participants with and without a current 12-month internalizing disorder (Additional file 1: Table S6-S10). Finally, the prevalence of specific phobia, comorbid disorders, and suicidality were all lower in low- or middle-income countries compared to high-income countries; however, the associations between number of SP subtypes and comorbidity or suicidality were similar regardless of country income level (Additional file 1: Table S11-16).

\section{Discussion}

\section{Principal findings}

In this study, we used a large cross-national sample to explore the associations of childhood generalized SP

Table 4 Age of onset of comorbid disorders, as a function of the number of specific phobia subtypes

\begin{tabular}{|c|c|c|c|c|c|c|c|c|c|}
\hline \multirow[t]{3}{*}{ Comorbid disorder } & \multicolumn{5}{|c|}{ Number of subtypes } & \multirow{2}{*}{\multicolumn{2}{|c|}{$\begin{array}{l}\text { Test of linear effect } \\
\text { (whole sample) }\end{array}$}} & \multirow{2}{*}{\multicolumn{2}{|c|}{$\begin{array}{l}\text { Test of linear effect } \\
\text { (SP cases only) }\end{array}$}} \\
\hline & \multirow{2}{*}{$\begin{array}{l}0 \\
\text { Median (IQR) }\end{array}$} & \multirow{2}{*}{1} & \multirow{2}{*}{$\begin{array}{l} \\
\text { Median (IQR) }\end{array}$} & \multirow{2}{*}{$\begin{array}{l}3 \\
\text { Median (IQR) }\end{array}$} & \multirow{2}{*}{$\begin{array}{l}+ \\
\text { Median (IQR) }\end{array}$} & & & & \\
\hline & & & & & & $\mathrm{OR}(95 \% \mathrm{Cl})$ & $p$ value & OR $(95 \% \mathrm{Cl})$ & $P$ valu \\
\hline Agoraphobia & $21(13-35)$ & $21(13-39)$ & $20(13-40)$ & $16(8-31)$ & $12(8-17)$ & $1.2^{*}(1.1-1.2)$ & $<0.001$ & $1.3^{*}(1.2-1.4)$ & $<0.001$ \\
\hline eneralized anxiety disorder & $39(26-54)$ & $37(24-52)$ & $35(21-52)$ & $33(20-44)$ & $28(18-46)$ & $1.2^{*}(1.1-1.2)$ & $<0.001$ & $1.2^{*}(1.1-1.2)$ & $<0.00$ \\
\hline Panic disorder & $33(21-49)$ & $29(19-41)$ & $35(18-60)$ & $24(14-41)$ & $18(11-27)$ & $1.2^{*}(1.2-1.3)$ & $<0.001$ & $1.2^{*}(1.1-1.3)$ & $<0.00$ \\
\hline Post-traumatic stress disorder & $36(21-54)$ & $33(20-49)$ & $36(19-53)$ & $35(19-75)$ & $33(19-64)$ & $1.1^{*}(1.0-1.1)$ & 0.002 & $1.1(1.0-1.2)$ & 0.166 \\
\hline Separation anxiety disorder & $19(10-31)$ & $19(8-29)$ & $18(8-30)$ & $14(8-36)$ & $17(8-28)$ & $1.0(1.0-1.1)$ & 0.074 & $1.0(1.0-1.1)$ & 0.235 \\
\hline Social anxiety disorder & $14(9-18)$ & $13(10-16)$ & $13(8-16)$ & $13(8-17)$ & $12(7-15)$ & $1.2^{*}(1.1-1.2)$ & $<0.001$ & $1.3^{*}(1.3-1.4)$ & $<0.00$ \\
\hline Major depression/dysthymia & $38(25-53)$ & $33(22-48)$ & $34(21-51)$ & $30(18-50)$ & $29(15-46)$ & $1.2^{*}(1.1-1.2)$ & $<0.001$ & $1.1^{*}(1.0-1.2)$ & 0.001 \\
\hline Bipolar disorder & $25(18-38)$ & $26(18-44)$ & $28(19-45)$ & $33(20-45)$ & $24(18-32)$ & $1.0(1.0-1.1)$ & 0.169 & $1.2^{*}(1.1-1.3)$ & 0.001 \\
\hline
\end{tabular}

Median age of onset was determined using the actuarial method. Differences in age of onset were tested with a discrete-time logistic regression, with the sample limited to participants who developed the comorbid disorder, and with number of early phobia subtypes time-varying until age 13 ${ }^{*} p<0.005$ 
Table 5 Days out of role, suicidality, and serious mental illness

\begin{tabular}{|c|c|c|c|c|c|c|c|c|c|c|}
\hline \multirow[t]{3}{*}{ Category } & \multirow[t]{3}{*}{ Subcategory } & \multicolumn{5}{|c|}{ Number of subtypes } & \multirow{2}{*}{\multicolumn{2}{|c|}{$\begin{array}{l}\text { Test of linear effect } \\
\text { (total sample) }\end{array}$}} & \multirow{2}{*}{\multicolumn{2}{|c|}{$\begin{array}{l}\text { Test of linear effect } \\
\text { (SP cases only) }\end{array}$}} \\
\hline & & \multirow{2}{*}{$\begin{array}{l}0 \\
\text { Mean } \\
(\mathrm{SE})\end{array}$} & \multirow{2}{*}{$\begin{array}{l}1 \\
\begin{array}{l}\text { Mean } \\
\text { (SE) }\end{array}\end{array}$} & \multirow{2}{*}{$\begin{array}{l} \\
\begin{array}{l}\text { Mean } \\
\text { (SE) }\end{array}\end{array}$} & \multirow{2}{*}{$\begin{array}{l}3 \\
\text { Mean } \\
(\mathrm{SE})\end{array}$} & \multirow{2}{*}{$\begin{array}{l}+ \\
\text { Mean } \\
\text { (SE) }\end{array}$} & & & & \\
\hline & & & & & & & $B(\mathrm{SE})$ & $\begin{array}{l}p \\
\text { value }\end{array}$ & $B(\mathrm{SE})$ & $\begin{array}{l}p \\
\text { value }\end{array}$ \\
\hline \multirow[t]{2}{*}{$\begin{array}{l}\text { Days out of role in the past } 30 \\
\text { days }\end{array}$} & & $\begin{array}{l}1.05 \\
(0.03)\end{array}$ & $\begin{array}{l}1.80 \\
(0.19)\end{array}$ & $\begin{array}{l}1.94 \\
(0.17)\end{array}$ & $\begin{array}{l}2.51 \\
(0.30)\end{array}$ & $\begin{array}{l}3.53 \\
(0.53)\end{array}$ & $0.56^{*}(0.06)$ & $\begin{array}{l}< \\
0.001\end{array}$ & $0.49^{*}(0.15)$ & $\begin{array}{l}< \\
0.001\end{array}$ \\
\hline & & $\%(S E)$ & $\%(\mathrm{SE})$ & $\%(S E)$ & $\%(S E)$ & $\%(\mathrm{SE})$ & OR (95\% Cl) & $\begin{array}{l}p \\
\text { value }\end{array}$ & OR $(95 \% \mathrm{Cl})$ & $\begin{array}{l}p \\
\text { value }\end{array}$ \\
\hline \multirow[t]{3}{*}{ Suicidality } & Ideation & $7.5(0.1)$ & $18.8(0.8)$ & $23.7(1.3)$ & $30.7(2.0)$ & $31.8(2.4)$ & $\begin{array}{l}1.6^{*}(1.6- \\
1.7)\end{array}$ & $\begin{array}{l}< \\
0.001\end{array}$ & $\begin{array}{l}1.3^{*}(1.2- \\
1.3)\end{array}$ & $\begin{array}{l}< \\
0.001\end{array}$ \\
\hline & Plan & $2.3(0.1)$ & $6.7(0.5)$ & $9.5(0.8)$ & $13.6(1.4)$ & $14.1(1.8)$ & $\begin{array}{l}1.7^{*}(1.6- \\
1.8)\end{array}$ & $\begin{array}{l}< \\
0.001\end{array}$ & $\begin{array}{l}1.3^{*}(1.2- \\
1.4)\end{array}$ & $\begin{array}{l}< \\
0.001\end{array}$ \\
\hline & Attempt & $2.0(0.0)$ & $6.5(0.4)$ & $8.4(0.7)$ & $13.4(1.3)$ & $16.8(1.7)$ & $\begin{array}{l}1.7^{*}(1.7- \\
1.8)\end{array}$ & $\begin{array}{l}< \\
0.001\end{array}$ & $\begin{array}{l}1.4^{*}(1.3- \\
1.5)\end{array}$ & $\begin{array}{l}< \\
0.001\end{array}$ \\
\hline Serious mental illness & & $3.5(0.1)$ & $12.7(0.7)$ & $20.9(1.2)$ & $27.8(2.1)$ & $36.8(2.7)$ & $\begin{array}{l}2.1^{*}(2.0- \\
2.2)\end{array}$ & $\begin{array}{l}< \\
0.001\end{array}$ & $\begin{array}{l}1.5^{*}(1.4- \\
1.7)\end{array}$ & $\begin{array}{l}< \\
0.001\end{array}$ \\
\hline
\end{tabular}

${ }^{*} p<0.005$

with the prevalence, persistence, and severity of other internalizing disorders. Among the $7.6 \%$ of participants reporting lifetime SP, most (78\%) had already developed the disorder before age 13. Childhood SP was highly persistent, although severe disability and treatment-seeking for SP were uncommon. Many respondents with childhood SP reported multiple phobias (44\%), with $8 \%$ even reporting four or more phobias. Childhood SP was strongly associated with prevalence, persistence, and severity of other internalizing disorders as well as with an early age of onset of these disorders. It was also associated with increased number of days out of role and SMI, as well as with suicidality. At a population level, 8.7$38.8 \%$ of all internalizing disorders and $13.9-20.4 \%$ of all suicidality or SMI were associated with childhood SP. Furthermore, associations persisted throughout the lifespan. Of particular importance, participants with generalized specific phobia had much worse outcomes than those with a single fear. For example, 17\% of those with four or more phobias reported a suicide attempt, compared to only $7 \%$ of those with a single phobia.

$\mathrm{SP}$ is generally viewed as a relatively mild disorder that causes less disability than other mood or anxiety disorders [24]. However, our results suggest that childhood SP, particularly when generalized, is strongly associated with poor long-term outcomes in the internalizing domain. Caution is needed since these results are based on a retrospective cohort design and recall error could lead to bias. However, the stability of the results across the age range of this very large and diverse sample suggests that the pattern is worthy of future investigation.

The present study is a practical application of suggestions made in the Hierarchical Taxonomy Of Psychopathology (HiTOP) initiative, which aims to develop an empirically driven nosology of psychopathology [44].
One fundamental aspect of HiTOP is its reliance on quantitative, rather than dichotomous measures, to more closely approximate the true nature of psychopathology. Although we have examined a specific disorder, we have used a more quantitative approach than the strictly DSM approach by looking at the number of SP subtypes. Furthermore, we chose to focus on specific phobia because it tends to be the earliest manifestation of an internalizing liability, not because we think it is unique. The results suggest that it might be possible to identify at least some of the people with a strong internalizing liability in childhood even though their childhood disorders typically are not severe.

A similar approach could be applied to other psychopathology domains. For instance, the externalizing domain includes substance use disorders, which usually first appear in late adolescence, as well as disorders with childhood onset, such as attention deficit hyperactivity disorder (ADHD) [3]. For other domains, such as thought disorders, subclinical psychotic experiences or personality traits like suspiciousness might be relevant $[45,46]$. Examination of personality traits might also help refine the early identification of persons with high internalizing or externalizing liabilities.

From the point of view of prevention, one might argue whether targeted interventions should be aimed at everyone with childhood SP or at a smaller or larger group. If resources are limited, targeting those with multiple phobias is reasonable, as the tendency to generalize fear appears to predict a particularly poor prognosis. For instance, reducing the risk for just this very small group $(0.5 \%)$ to that of people without childhood SP could prevent $1.1-7.8 \%$ of other internalizing disorders and 3.5\% of all suicide attempts. However, even participants with a single phobia had increased risks of unfavorable 
outcomes. The population attributable fraction for suicide attempts for all childhood SP, for instance, was $20.4 \%$, showing that much greater benefits could be obtained by targeting this larger group. Furthermore, previous research has found that most children reporting a specific fear do not meet SP criteria [31]. It is currently unclear whether such fearfulness is harmless or a sign of an internalizing vulnerability, for instance when fearfulness is generalized.

As our study is observational, we cannot establish causality. We hypothesize that the associations are not directly causal, but that both childhood SP and later outcomes are expressions of a latent internalizing vulnerability. Although SP is a promising target in part because it is a relatively easy to treat disorder [47] that nevertheless often remains untreated, it is unclear whether treatment specifically for SP would have substantial effects on internalizing outcomes later in life. Exposure is a common element in cognitive behavioral therapy, so broader effects on the underlying vulnerability are plausible, and there is some evidence to suggest that treatment for phobia can lead to improvement in already-existing comorbid anxiety disorders [48]. Since even single-session behavioral therapy can be sufficient for SP [49], and since up to $20 \%$ of suicide attempts and $19 \%$ of SMI are attributable to childhood SP (assuming a causal relationship), it would be highly worthwhile to examine whether early treatment of specific phobia has a substantial effect on these other outcomes.

In contrast to most previous research regarding SP, which used participant samples from high-income Western countries, our sample included a diverse set of countries. We found that both SP and our main outcomes, comorbidity and suicidality, were less prevalent in lowand middle-income countries than in high-income countries. However, the associations between childhood (generalized) SP and these outcomes were remarkably similar across country income levels. This suggests that childhood generalized SP might be a globally useful and not culturally specific marker for at-risk children. However, treatment rates for childhood-onset SP are even lower in low- or middle-income countries $(12.7 \%)$ than in high-income countries (26.8\%), and it may be difficult to detect and provide early intervention to these children if resources are scarce. Ideally, to reach as many people as possible, any screening or intervention program for childhood generalized SP should also be feasible in countries with relatively few specialist mental health care providers.

\section{Strengths and limitations}

The World Mental Health Surveys provide a unique opportunity to examine childhood SP and its relationship to other internalizing disorders in a very large sample from a diverse set of countries using a common protocol and instrument. However, there are also several important limitations. First, data are derived from a cross-sectional interview of adult participants, and age of onset is therefore estimated retrospectively. While the survey was designed using modern cognitive interviewing methods as a way to encourage active memory search and improve recall accuracy [50], some recall bias doubtlessly persists. Mild SP may have been forgotten, so our sample may not be fully representative of all children with SP. However, while some of our research questions could also be examined with existing longitudinal studies, no longitudinal studies with a sufficiently large sample size exists that could examine all the questions considered here. We also took several steps to investigate whether recall bias could explain our results. Firstly, we performed sensitivity analyses in the separate age groups and found broadly similar results in the youngest age group (18-34 years old), for whom recall bias should be least problematic, compared to the oldest age group (50+ years old). Presence of current psychopathology could also lead to recall bias. If respondents with current psychopathology are more likely to recall symptoms they suffered previously, including specific phobias, this could lead to a spurious association between childhood SP and comorbidity. However, we also found strong associations between number of childhood SP subtypes and lifetime comorbidity within the subgroup of respondents that did not report a 12-month internalizing disorder. Secondly, the observational design precludes causal inferences. Finally, the CIDI does not assess all phobias. Consequently, we may have underestimated the number of childhood SP subtypes.

\section{Conclusions}

In conclusion, this study has shown that childhood generalized SP, as assessed in a retrospective survey, is strongly associated with poor outcomes in the internalizing domain of psychopathology throughout the life course. While our study cannot establish whether childhood SP is causally associated with these later outcomes or whether some other factor, such as an underlying internalizing vulnerability, explains the association, it clearly identifies children with generalized SP as a high-risk group. Respondents with childhood SP not only were more likely than other respondents to develop internalizing disorders, but developed them at an earlier age and had a more persistent and severe course, including more disability, suicidality, and serious mental illness. Although even respondents with a single childhood SP subtype had an elevated risk of poor outcomes, risk was much higher among respondents who reported multiple childhood SP subtypes. Children with generalized SP might therefore be an important target group for early intervention to reduce internalizing psychopathology across the lifespan. 


\section{Additional file}

Additional file 1: Supplemental tables providing additional information. (DOCX $52 \mathrm{~kb}$ )

\section{Abbreviations}

ADHD: Attention deficit hyperactivity disorder; Cl: Confidence interval; CIDI: Composite International Diagnostic Interview; DSM: Diagnostic and Statistical Manual of Mental Disorders; GAD: Generalized anxiety disorder; HiTOP: Hierarchical Taxonomy Of Psychopathology; IQR: Interquartile range; MDD: Major depressive disorder; OR: Odds ratio; PAF: Population attributable fraction; PTSD: Post-traumatic stress disorder; SCID: Structured Clinical Interview for DSM-IV; SD: Standard deviation; SDS: Sheehan Disability Scale; SE: Standard error; SMI: Serious mental illness; SP: Specific phobia; WMHS: World Mental Health Surveys

\section{Acknowledgements}

Group information: The WHO World Mental Health Survey collaborators are Sergio Aguilar-Gaxiola, MD, PhD; Ali Al-Hamzawi, MD; Mohammed Salih AlKaisy, MD; Jordi Alonso, MD, PhD; Laura Helena Andrade, MD, PhD; Corina Benjet, PhD; Guilherme Borges, ScD; Evelyn J. Bromet, PhD; Ronny Bruffaerts, PhD; Brendan Bunting, PhD; Jose Miguel Caldas de Almeida, MD, PhD; Graça Cardoso, MD, PhD; Somnath Chatterji, MD; Alfredo H. Cia, MD; Louisa Degenhardt, PhD; Koen Demyttenaere, MD, PhD; Silvia Florescu, MD, PhD; Giovanni de Girolamo, MD; Oye Gureje, MD, DSc, FRCPsych; Josep Maria Haro, MD, PhD; Hristo Hinkov, MD, PhD; Chi-yi Hu, MD, PhD; Peter de Jonge, PhD; Aimee Nasser Karam, PhD; Elie G. Karam, MD; Norito Kawakami, MD, DMSc; Ronald C. Kessler, PhD; Andrzej Kiejna, MD, PhD; Viviane Kovess-Masfety, MD, PhD; Sing Lee, MB, BS; Jean-Pierre Lepine, MD; Daphna Levinson, PhD; John McGrath, MD, PhD; Maria Elena Medina-Mora, PhD; Zeina Mneimneh, PhD; Jacek Moskalewicz, PhD; Fernando Navarro-Mateu, MD, PhD; Beth-Ellen Pennell, MA; Marina Piazza, MPH, ScD; Jose Posada-Villa, MD; Kate M. Scott, PhD; Tim Slade, PhD; Juan Carlos Stagnaro, MD, PhD; Dan J. Stein, FRCPC, PhD; Margreet ten Have, PhD; Yolanda Torres, MPH, Dra.HC; Maria Carmen Viana, MD, PhD; Harvey Whiteford, MBBS, PhD; David R. Williams, MPH, PhD; and Bogdan Wojtyniak, ScD.

\section{Funding}

The World Health Organization World Mental Health (WMH) Survey Initiative is supported by the US National Institute of Mental Health (NIMH; R01 MH070884), the John D. and Catherine T. MacArthur Foundation, the Pfizer Foundation, the United States Public Health Service (R13-MH066849, R01MH069864, and R01 DA016558), the Fogarty International Center (FIRCA R03TW006481), the Pan American Health Organization, Eli Lilly and Company, Ortho-McNeil Pharmaceutical Inc., GlaxoSmithKline, and Bristol-Myers Squibb. We thank the staff of the WMH Data Collection and Data Analysis Coordination Centres for the assistance with instrumentation, fieldwork, and consultation on data analysis. None of the funders had any role in the design, analysis, interpretation of results, or preparation of this paper. The views and opinions expressed in this report are those of the authors and should not be construed to represent the views of the World Health Organization, other sponsoring organizations, agencies, or governments.

The Argentina survey-Estudio Argentino de Epidemiología en Salud Mental (EASM) - was supported by a grant from the Argentinian Ministry of Health (Ministerio de Salud de la Nación). The São Paulo Megacity Mental Health Survey is supported by the State of São Paulo Research Foundation (FAPESP) Thematic Project Grant 03/00204-3. The Bulgarian Epidemiological Study of common mental disorders EPIBUL is supported by the Ministry of Health and the National Center for Public Health Protection. The Chinese World Mental Health Survey Initiative is supported by the Pfizer Foundation. The Colombian National Study of Mental Health (NSMH) is supported by the Ministry of Social Protection. The Mental Health Study Medellín-Colombia was carried out and supported jointly by the Center for Excellence on Research in Mental Health (CES University) and the Secretary of Health of Medellín. The ESEMeD project is funded by the European Commission (Contracts QLG5-1999-01042; SANCO 2004123, and EAHC 20081308), the Piedmont Region (Italy), Fondo de Investigación Sanitaria, Instituto de Salud Carlos III, Spain (FIS 00/0028), Ministerio de Ciencia y Tecnología, Spain (SAF 2000-158-CE), Departament de Salut, Generalitat de Catalunya, Spain, DIUE de la Generalitat de Catalunya (2017 SGR 452; 2014 SGR 748), Instituto de
Salud Carlos III (CIBER CB06/02/0046, RETICS RD06/0011 REM-TAP), and other local agencies and by an unrestricted educational grant from GlaxoSmithKline. Implementation of the Iraq Mental Health Survey (IMHS) and data entry were carried out by the staff of the Iraqi $\mathrm{MOH}$ and MOP with direct support from the Iraqi IMHS team with funding from both the Japanese and European Funds through United Nations Development Group Iraq Trust Fund (UNDG ITF). The World Mental Health Japan (WMHJ) Survey is supported by the Grant for Research on Psychiatric and Neurological Diseases and Mental Health (H13SHOGAI-023, H14-TOKUBETSU-026, H16-KOKORO-013, H25-SEISHIN-IPPAN-006) from the Japan Ministry of Health, Labour and Welfare. The Lebanese Evaluation of the Burden of Ailments and Needs Of the Nation (L.E.B.A.N.O.N.) is supported by the Lebanese Ministry of Public Health, the WHO (Lebanon), National Institute of Health/Fogarty International Center (R03 TW006481-01); anonymous private donations to IDRAAC, Lebanon; and unrestricted grants from, Algorithm, AstraZeneca, Benta, Bella Pharma, Eli Lilly, Glaxo Smith Kline, Lundbeck, Novartis, OmniPharma, Pfizer, Phenicia, Servier, and UPO. The Mexican National Comorbidity Survey (MNCS) is supported by The National Institute of Psychiatry Ramon de la Fuente (INPRFMDIES 4280) and by the National Council on Science and Technology (CONACYT-G30544- H), with supplemental support from the Pan American Health Organization (PAHO). Te Rau Hinengaro: The New Zealand Mental Health Survey (NZMHS) is supported by the New Zealand Ministry of Health, Alcohol Advisory Council, and the Health Research Council. The Nigerian Survey of Mental Health and Wellbeing (NSMHW) is supported by the WHO (Geneva), the WHO (Nigeria), and the Federal Ministry of Health, Abuja, Nigeria. The Northern Ireland Study of Mental Health was funded by the Health \& Social Care Research \& Development Division of the Public Health Agency. The Peruvian World Mental Health Study was funded by the National Institute of Health of the Ministry of Health of Peru. The Polish project Epidemiology of Mental Health and Access to Care-EZOP Project (PL 0256) was supported by Iceland, Liechtenstein, and Norway through funding from the EEA Financial Mechanism and the Norwegian Financial Mechanism. EZOP project was co-financed by the Polish Ministry of Health. The Portuguese Mental Health Study was carried out by the Department of Mental Health, Faculty of Medical Sciences, NOVA University of Lisbon, with collaboration of the Portuguese Catholic University, and was funded by Champalimaud Foundation, Gulbenkian Foundation, Foundation for Science and Technology (FCT) and Ministry of Health. The Romania WMH study projects "Policies in Mental Health Area" and "National Study regarding Mental Health and Services Use" were carried out by the National School of Public Health \& Health Services Management (former National Institute for Research \& Development in Health), with technical support of Metro Media Transilvania, the National Institute of Statistics-National Centre for Training in Statistics, SC, Cheyenne Services SRL, Statistics Netherlands and were funded by the Ministry of Public Health (former Ministry of Health) with supplemental support of Eli Lilly Romania SRL. The Psychiatric Enquiry to General Population in Southeast Spain-Murcia (PEGASUSMurcia) Project has been financed by the Regional Health Authorities of Murcia (Servicio Murciano de Salud and Consejería de Sanidad y Política Social) and Fundación para la Formación e Investigación Sanitarias (FFIS) of Murcia. The US National Comorbidity Survey Replication (NCS-R) is supported by the National Institute of Mental Health (NIMH; U01-MH60220) with supplemental support from the National Institute of Drug Abuse (NIDA), the Substance Abuse and Mental Health Services Administration (SAMHSA), the Robert Wood Johnson Foundation (RWJF; Grant 044708), and the John W. Alden Trust.

A complete list of all within-country and cross-national WMH publications can be found at http://www.hcp.med.harvard.edu/wmh/.

\section{Availability of data and materials}

Access to the cross-national World Mental Health (WMH) data is governed by the organizations funding and responsible for survey data collection in each country. These organizations made data available to the WMH consortium through restricted data sharing agreements that do not allow us to release the data to third parties. The exception is that the US data are available for secondary analysis via the Inter-University Consortium for Political and Social Research (ICPSR) http://www.icpsr.umich.edu/icpsrweb/ICPSR/series/00527.

\section{Authors' contributions}

Y and PJ designed the study. YV performed the statistical analysis. $\mathrm{V}$ and PJ wrote the first draft of the manuscript. The other co-authors contributed to the data acquisition and interpretation and provided critical feedback on drafts of the manuscript. All authors read and approved the final version of the manuscript. 


\section{Ethics approval and consent to participate}

Local Institutional Review Boards approved each survey, and all respondents gave informed consent. Approval was granted by the Bioethics Committee of the School of Medicine, University of Buenos Aires, in Argentina; the Ethics Committee of the Institute of Public Health in Belgium; the Research and Ethics Committee of the School of Medicine, University of Sao Paulo, in Brazil; the Deputy Minister and head of the Executive Committee of the National Psychiatric Program in Bulgaria; the Ethics Committee for the FES Social Foundation in Colombia; the Comité Institucional de Ética Universidad CES in Colombia-Medellin; the Committee of the Commission Nationale Informatique et Libertés in France; the Ethics Committee of the University of Leipzig in Germany; the Ethics Scientific Committee of the Ministry of Health in Iraq; the Italian National Institute of Health in Italy; the National Center of Neurology and Psychiatry, Okayama University School of Medicine, Nagasaki University School of Medicine, Keio University School of Medicine, Jichi University School of Medicine, Juntendo University School of Medicine, and Yamagata University School of Medicine in Japan; the University of Balamand Faculty of Medicine Institutional Review Board in Lebanon; the Ethics Committee in research of the National Institute of Psychiatry Ramon de la Fuente Muñiz in Mexico; the Ethics Committee of the Netherlands Institute of Mental Health and Addiction in the Netherlands; the New Zealand Health Ethics Committees in New Zealand; the University of Ibadan and University College Hospital Join Ethics Committee in Nigeria; the University of Ulster Ethics Committee in Northern Ireland; the National Institute of Health in Peru; the Ethics Committee of the Shenzhen Kangning Hospital in Shenzhen; the Bioethical Committee of Instytut Psychiatrii i Neurologi in Poland; the Ethics Committee of the Faculdade de Ciencias Médicas, Universidade Nova, in Portugal; the Ethic Commission of the Scientific Board of National Institute for Research and Development in Health in Romania; the Ethical Committee of Sant Joan de Deu Serveis de Salut Menta and the Ethical Committee of IMIM-Hospital del Mar Medical Research Institute in Spain; the Clinical Research Ethics Committee of the University Hospital Virgen de la Arrixaca in SpainMurcia; and the Human Subjects Committees of the Institute for Social Research at the University of Michigan and of Harvard Medical School in the USA.

\section{Consent for publication}

Not applicable.

\section{Competing interests}

In the past 3 years, Dr. Kessler received support for his epidemiological studies from Sanofi Aventis; was a consultant for Johnson \& Johnson Wellness and Prevention, Sage Pharmaceuticals, Shire, Takeda; and served on an advisory board for the Johnson \& Johnson Services Inc. Lake Nona Life Project. Kessler is a co-owner of DataStat, Inc., a market research firm that carries out healthcare research. All other authors declare that they have competing interests.

\section{Publisher's Note}

Springer Nature remains neutral with regard to jurisdictional claims in published maps and institutional affiliations.

\footnotetext{
Author details

${ }^{1}$ Faculty of Behavioural and Social Sciences, Department of Developmental Psychology, University of Groningen, Groningen, the Netherlands. ${ }^{2}$ Interdisciplinary Center Psychopathology and Emotion regulation, University Medical Center Groningen, University of Groningen, Groningen, the Netherlands. ${ }^{3}$ College of Medicine, Al-Qadisiya University, Diwaniya governorate, Iraq. ${ }^{4}$ Health Services Research Unit, IMIM-Hospital del Mar Medical Research Institute, Barcelona, Spain. ${ }^{5}$ CIBER en Epidemiología y Salud Pública (CIBERESP), Barcelona, Spain. ${ }^{6}$ Pompeu Fabra University (UPF), Barcelona, Spain. ${ }^{7}$ National Institute of Psychiatry Ramón de la Fuente Muñiz, Mexico City, Mexico. ${ }^{8}$ Universitair Psychiatrisch Centrum - Katholieke Universiteit Leuven (UPC-KUL), Campus Gasthuisberg, Leuven, Belgium. ${ }^{9}$ School of Psychology, Ulster University, Londonderry, UK. ${ }^{10}$ Lisbon Institute of Global Mental Health and Chronic Diseases Research Center (CEDOC), NOVA Medical School / Faculdade de Ciências Médicas, Universidade Nova de Lisboa, Lisbon, Portugal. ${ }^{11}$ Anxiety Clinic and Research Center, Buenos Aires, Argentina. ${ }^{12}$ RCCS Istituto Centro San Giovanni di Dio Fatebenefratelli, Brescia, Italy. ${ }^{13}$ National Center of Public Health and Analyses, Sofia, Bulgaria. ${ }^{14}$ Department of Psychiatry, College of Medicine, University of Ibadan, Ibadan, Nigeria. ${ }^{15}$ National School of Public Health, Management and
}

Development, Bucharest, Romania. ${ }^{16}$ Department of Psychiatry, University College Hospital, Ibadan, Nigeria. ${ }^{17}$ Parc Sanitari Sant Joan de Déu, CIBERSAM, Universitat de Barcelona, Sant Boi de Llobregat, Barcelona, Spain. ${ }^{18}$ Department of Psychology, College of Education, King Saud University, Riyadh, Saudi Arabia. ${ }^{19}$ Shenzhen Institute of Mental Health and Shenzhen Kangning Hospital, Shenzhen, China. ${ }^{20}$ Department of Psychiatry and Clinical Psychology, Faculty of Medicine, Balamand University, Beirut, Lebanon. ${ }^{21}$ Department of Psychiatry and Clinical Psychology, St George Hospital University Medical Center, Beirut, Lebanon. ${ }^{22}$ Institute for Development Research Advocacy and Applied Care (IDRAAC), Beirut, Lebanon. ${ }^{23}$ National Institute of Mental Health, National Center for Neurology and Psychiatry, Kodaira, Tokyo, Japan. ${ }^{24}$ Wroclaw Medical University, Wrocław, Poland. ${ }^{25}$ University of Lower Silesia, Wroclaw, Poland. ${ }^{26}$ Ecole des Hautes Etudes en Santé Publique (EHESP), EA 4057, Paris Descartes University, Paris, France. ${ }^{27}$ Department of Psychiatry, Chinese University of Hong Kong, Tai Po, Hong Kong. ${ }^{28}$ Survey Research Center, Institute for Social Research, University of Michigan, Ann Arbor, MI, USA. ${ }^{29}$ UDIF-SM, Subdirección General de Planificación, Innovación y Cronicidad, Servicio Murciano de Salud, IMIB-Arrixaca, CIBERESP-Murcia, Murcia, Spain. ${ }^{30}$ Instituto Murciano de Investigación Biosanitaria (IMIB) Virgen de la Arrixaca, Murcia, Spain. ${ }^{31}$ Centro de Investigación Biomédica en ERed en Epidemiología y Salud Pública (CIBERESP), Murcia, Spain. ${ }^{32}$ Instituto Nacional de Salud, Lima, Peru. ${ }^{33}$ Universidad Cayetano Heredia, Lima, Peru. ${ }^{34}$ Department of Psychological Medicine, University of Otago, Dunedin, Otago, New Zealand. ${ }^{35}$ Trimbos Instituut, Netherlands Institute of Mental Health and Addiction, Utrecht, Netherlands. ${ }^{36}$ Center for Excellence on Research in Mental Health, CES University, Medellin, Colombia. ${ }^{37}$ Department of Social Medicine, Postgraduate Program in Public Health, Federal University of Espírito Santo, Vitoria, Brazil. ${ }^{38}$ Department of Health Care Policy, Harvard Medical School, Boston, MA, USA.

\section{Received: 23 January 2019 Accepted: 17 April 2019}

Published online: 24 May 2019

\section{References}

1. GBD 2015 Disease and Injury Incidence and Prevalence Collaborators. Global, regional, and national incidence, prevalence, and years lived with disability for 310 diseases and injuries, 1990-2015: a systematic analysis for the Global Burden of Disease Study 2015. Lancet. 2016;388:1545-602.

2. The WHO World Mental Health Survey Consortium. Prevalence, severity, and unmet need for treatment of mental disorders in the World Health Organization World Mental Health Surveys. JAMA. 2004;291:2581-90.

3. Kessler RC, Berglund PA, Demler O, Jin R, Merikangas KR, Walters EE. Lifetime prevalence and age-of-onset distributions of DSM-IV disorders in the National Comorbidity Survey Replication. Arch Gen Psychiatry. 2005;62:593-603.

4. Penninx BWJH, Nolen WA, Lamers F, Zitman FG, Smit JH, Spinhoven P, et al. Two-year course of depressive and anxiety disorders: results from the Netherlands Study of Depression and Anxiety (NESDA). J Affect Disord. 2011;133:76-85.

5. Bruce SE, Yonkers KA, Otto MW, Eisen JL, Weisberg RB, Pagano M, et al. Influence of psychiatric comorbidity on recovery and recurrence in generalized anxiety disorder, social phobia, and panic disorder: a 12-year prospective study. Am J Psychiatry. 2005;162:1179-87.

6. Judd LL, Akiskal HS, Maser JD, Zeller PJ, Endicott J, Coryell W, et al. A prospective 12-year study of subsyndromal and syndromal depressive symptoms in unipolar major depressive disorders. Arch Gen Psychiatry. 1998:55:694-700.

7. Turner EH, Matthews AM, Linardatos E, Tell RA, Rosenthal R. Selective publication of antidepressant trials and its influence on apparent efficacy. $N$ Engl J Med. 2008;358:252-60.

8. Roest AM, de Jonge P, Williams CD, de Vries YA, Schoevers RA, Turner EH. Reporting bias in clinical trials investigating the efficacy of secondgeneration antidepressants in the treatment of anxiety disorders: a report of 2 meta-analyses. JAMA Psychiatry. 2015;72:500-10.

9. Huhn M, Tardy M, Spineli LM, Kissling W, Förstl H, Pitschel-Walz G, et al. Efficacy of pharmacotherapy and psychotherapy for adult psychiatric disorders: a systematic overview of meta-analyses. JAMA Psychiatry. 2014;71:706.

10. Andrews G, Sanderson K, Corry J, Lapsley HM. Using epidemiological data to model efficiency in reducing the burden of depression. J Ment Health Policy Econ. 2000;3:175-86. 
11. Chisholm D, Sanderson K, Ayuso-Mateos JL, Saxena S, C D, S K, et al. Reducing the global burden of depression: population-level analysis of intervention costeffectiveness in 14 world regions. Br J Psychiatry. 2004;184:393-403.

12. Kessler RC, Chiu WT, Demler O, Walters EE. Prevalence, severity, and comorbidity of 12-month DSM-IV disorders in the National Comorbidity Survey Replication. Arch Gen Psychiatry. 2005;62:617-27.

13. Ormel J, Raven D, van Oort F, Hartman CA, Reijneveld SA, Veenstra R, et al. Mental health in Dutch adolescents: a TRAILS report on prevalence, severity, age of onset, continuity and co-morbidity of DSM disorders. Psychol Med. 2015:45:345-60.

14. Krueger RF. The structure of common mental disorders. Arch Gen Psychiatry. 1999:56:921-6.

15. de Jonge P, Wardenaar KJ, Lim CCW, Aguilar-Gaxiola S, Alonso J, Andrade $\mathrm{LH}$, et al. The cross-national structure of mental disorders: results from the World Mental Health Surveys. Psychol Med. 2018;48:2073-84.

16. Kessler RC, Cox BJ, Green JG, Ormel J, McLaughlin KA, Merikangas KR, et al. The effects of latent variables in the development of comorbidity among common mental disorders. Depress Anxiety. 2011;28:29-39.

17. Markon KE. Modeling psychopathology structure: a symptom-level analysis of Axis I and II disorders. Psychol Med. 2010;40:273-88.

18. Eaton NR, Keyes KM, Krueger RF, Balsis S, Skodol AE, Markon KE, et al. An invariant dimensional liability model of gender differences in mental disorder prevalence: evidence from a national sample. J Abnorm Psychol. 2012;121:282-8.

19. Forbush KT, Watson D. The structure of common and uncommon mental disorders. Psychol Med. 2013;43:97-108.

20. Kessler RC, Ormel J, Petukhova M, McLaughlin KA, Green JG, Russo L, et al. Development of lifetime comorbidity in the World Health Organization world mental health surveys. Arch Gen Psychiatry. 2011;68:90-100.

21. Wardenaar KJ, Lim CCW, Al-Hamzawi AO, Alonso J, Andrade LH, Benjet C, et al. The cross-national epidemiology of specific phobia in the World Mental Health Surveys. Psychol Med. 2017;47:1744-60.

22. Scott KM, de Jonge P, Stein DJ, Kessler RC, editors. Mental disorders around the world: facts and figures from the WHO World Mental Health Surveys. 1. Cambridge: Cambridge University Press; 2018.

23. Comer JS, Blanco C, Hasin DS, Liu S-M, Grant BF, Turner B, et al. Health-related quality of life across the anxiety disorders. J Clin Psychiatry. 2011;72:43-50.

24. Ormel J, Petukhova M, Chatterji S, Aguilar-Gaxiola S, Alonso J, Angermeyer MC, et al. Disability and treatment of specific mental and physical disorders across the world. Br J Psychiatry. 2008;192:368-75.

25. Lieb R, Miche M, Gloster AT, Beesdo-Baum K, Meyer AH, Wittchen H-U. Impact of specific phobia on the risk of onset of mental disorders: a 10-year prospective-longitudinal community study of adolescents and young adults. Depress Anxiety. 2016;675:667-75

26. Depla MFIA, ten Have ML, van Balkom AJLM, de Graaf R. Specific fears and phobias in the general population: results from the Netherlands Mental Health Survey and Incidence Study (NEMESIS). Soc Psychiatry Psychiatr Epidemiol. 2008;43:200-8.

27. Goodwin RD. Anxiety disorders and the onset of depression among adults in the community. Psychol Med. 2002;32:S0033291702005482.

28. Magee WJ, Eaton WW, Wittchen HU, McGonagle KA, Kessler RC. Agoraphobia, simple phobia, and social phobia in the National Comorbidity Survey. Arch Gen Psychiatry. 1996;53:159-68.

29. Park S, Sohn JH, Hong JP, Chang SM, Lee YM, Jeon HJ, et al. Prevalence, correlates, and comorbidities of four DSM-IV specific phobia subtypes: results from the Korean Epidemiological Catchment Area study. Psychiatry Res. 2013;209:596-603.

30. Chou K-L. Specific phobia in older adults: evidence from the National Epidemiologic Survey on Alcohol and Related Conditions. Am J Geriatr Psychiatry. 2009;17:376-86.

31. Benjet C, Borges G, Stein DJ, Mendez E, Medina-Mora ME. Epidemiology of fears and specific phobia in adolescence: results from the Mexican Adolescent Mental Health Survey. J Clin Psychiatry. 2012;73:152-8.

32. Choy Y, Fyer AJ, Goodwin RD. Specific phobia and comorbid depression: a closer look at the National Comorbidity Survey data. Compr Psychiatry. 2007;48:132-6.

33. Stinson FS, D a D, Patricia Chou S, Smith S, Goldstein RB, June Ruan W, et al. The epidemiology of DSM-IV specific phobia in the USA: results from the National Epidemiologic Survey on Alcohol and Related Conditions. Psychol Med. 2007;37(March):1047-59.

34. Trumpf J, Margraf J, Vriends N, Meyer AH, Becker ES. Predictors of specific phobia in young women: a prospective community study. J Anxiety Disord. 2010;24:87-93.
35. Curtis GC, Magee WJ, Eaton WW, Wittchen HU, Kessler RC. Specific fears and phobias. Epidemiology and classification. Br J Psychiatry. 1998;173:212-7.

36. Burstein M, Georgiades K, He JP, Schmitz A, Feig E, Khazanov GK, et al. Specific phobia among U.S. adolescents: phenomenology and typology. Depress Anxiety. 2012;29:1072-82.

37. Heeringa SG, Wells JE, Hubbard F, Mneimneh Z, Chiu WT, Sampson N, et al. Sample designs and sampling procedures. In: Kessler RC, Üstün TB, editors. The WHO World Mental Health Surveys: global perspectives on the epidemiology of mental disorders. New York: Cambridge University Press; 2008. p. 14-32.

38. Pennell B, Mneimneh Z, Bowers A, Chardoul S, Wells JE, Viana MC, et al. Implementation of the world mental health surveys. In: Kessler RC, Üstün TB, editors. The WHO World Mental Health Surveys: global perspectives on the epidemiology of mental disorders. New York: Cambridge University Press; 2008. p. 33-57.

39. Kessler RC, Üstün BB. The World Mental Health (WMH) Survey Initiative version of the World Health Organization (WHO) Composite International Diagnostic Interview (CIDI). Int J Methods Psychiatr Res. 2004;13:93-117.

40. Haro JM, Arbabzadeh-Bouchez S, Brugha TS, De Girolamo G, Guyer ME, Jin $\mathrm{R}$, et al. Concordance of the Composite International Diagnostic Interview version 3.0 (CIDI 3.0) with standardized clinical assessments in the WHO World Mental Health Surveys. Int J Methods Psychiatr Res. 2006;15:167-80.

41. Sheehan DV, Harnett-Sheehan K, Raj BA. The measurement of disability. Int Clin Psychopharmacol. 1996;11(Supplement 3):89-95.

42. Levinson D, Lakoma MD, Petukhova M, Schoenbaum M, Zaslavsky AM, Angermeyer $\mathrm{M}$, et al. Associations of serious mental illness with earnings: results from the WHO World Mental Health surveys. Br J Psychiatry. 2010; 197:114-21.

43. Heeringa SG, Berglund PA, West BT, Mellipilán ER, Portier K. Attributable fraction estimation from complex sample survey data. Ann Epidemiol. 2015; 25:174-8

44. Kotov R, Krueger RF, Watson D, Achenbach TM, Althoff RR, Bagby RM, et al. The Hierarchical Taxonomy of Psychopathology (HiTOP): a dimensional alternative to traditional nosologies. J Abnorm Psychol. 2017;126:454-77.

45. Kaymaz N, Drukker M, Lieb R, Wittchen H-U, Werbeloff N, Weiser M, et al. Do subthreshold psychotic experiences predict clinical outcomes in unselected non-help-seeking population-based samples? A systematic review and meta-analysis, enriched with new results. Psychol Med. 2012;42:2239-53.

46. Poulton R, Caspi A, Moffitt TE, Cannon M. Children's self-reported psychotic symptoms and adult schizophreniform disorder. Arch Gen Psychiatry. 2000; $57: 7-12$.

47. Choy Y, Fyer AJ, Lipsitz JD. Treatment of specific phobia in adults. Clin Psychol Rev. 2007;27:266-86.

48. Ryan SM, Strege MV, Oar EL, Ollendick TH. One session treatment for specific phobias in children: comorbid anxiety disorders and treatment outcome. J Behav Ther Exp Psychiatry. 2017;54:128-34.

49. Zlomke K, Davis TE 3rd. One-session treatment of specific phobias: a detailed description and review of treatment efficacy. Behav Ther. 2008;39:207-23.

50. Knäuper B, Cannell C, Schwarz N, Bruce M, Kessler RC. Improving the accuracy of major depression age of onset reports in the US National Comorbidity Survey. Int J Methods Psychiatr Res. 1999:8:39-48.

\section{Ready to submit your research? Choose BMC and benefit from:}

- fast, convenient online submission

- thorough peer review by experienced researchers in your field

- rapid publication on acceptance

- support for research data, including large and complex data types

- gold Open Access which fosters wider collaboration and increased citations

- maximum visibility for your research: over $100 \mathrm{M}$ website views per year

At $\mathrm{BMC}$, research is always in progress.

Learn more biomedcentral.com/submissions 